Bull. Austral. Math. Soc.

VOL. 38 (1988) [207-220]

\title{
ON MINIMAL FAITHFUL PERMUTATION REPRESENTATIONS OF FINITE GROUPS.
}

\author{
David Easdown and Cheryl E. Praeger
}

\begin{abstract}
The minimal (faithful) degree $\mu(G)$ of a finite group $G$ is the least positive integer $n$ such that $G<S_{n}$. Clearly if $H \leqslant G$ then $\mu(H) \leqslant \mu(G)$. However if $N \triangleleft G$ then it is possible for $\tilde{\mu}(G / N)$ to be greater than $\mu(G)$; such groups $G$ are here called exceptional. Properties of exceptional groups are investigated and several families of exceptional groups are given. For example it is shown that the smallest exceptional groups have order 32 .
\end{abstract}

\section{INTRODUCTION}

The minimal (faithful) degree $\mu(G)$ of a finite group is the least positive integer $n$ such that $G$ can be embedded in $S_{n}$, the symmetric group on a set of size $n$, and any such embedding is called a minimal (faithful) representation of $G$. It is part of the folklore that $\mu(G)$ is the least value of $\sum_{1 \leqslant i \leqslant t}\left|G: H_{i}\right|$ where $H_{1}, \ldots, H_{t}$ are subgroups of $G$ for which $\bigcap_{1 \leqslant i \leqslant t} \bigcap_{g \in G} H_{i}^{g}=1$.

Though we do not consider semigroups which are not groups, one may define the minimal (faithful) degree $\mu(S)$ of a finite semigroup $S$ to be the least nonnegative integer $n$ such that $S$ can be embedded in $P T_{I} \times P T_{J}^{*}$, the direct product of a semigroup and dual semigroup of partial mappings on disjoint sets $I$ and $J$ respectively, where $I \cup J$ contains $n$ elements. It is easy to show this definition is equivalent to the earlier one when $S$ is a nontrivial group. Minimal degrees of semigroups which are not groups have been studied by one of the authors in $[1,2]$, and $[3]$.

In what follows $G$ will always denote a nontrivial finite group. Clearly $\mu(H) \leqslant$ $\mu(G)$ if $H \leqslant G$. However if $N \triangleleft G$ then it is possible for $\mu(G / N)$ to be greater than $\mu(G)$; in such a case $G$ will be called exceptional and we call $N$ and $G / N$ a distinguished subgroup and quotient respectively of $G$. This paper is the result of our investigations of exceptional groups and their distinguished subgroups and quotients.

We remark that if $G$ is abelian, so $G \simeq \mathbb{Z}_{p_{1} \alpha_{1}} \times \ldots \times \mathbb{Z}_{p_{n} \alpha_{n}}$ for some primes $p_{1}, \ldots, p_{n}$ and positive integers $\alpha_{1}, \ldots, \alpha_{n}$, then by $[\mathbf{7}, \mathbf{6}]$ we have $\mu(G)=\sum_{1 \leqslant i \leqslant n} p_{i}^{\alpha_{i}}$. An abelian group cannot be exceptional since each quotient is isomorphic to a subgroup.

Received 6 November, 1987

Copyright Clearance Centre, Inc. Serial-fee code: 0004-9729/88 \$A2.00+0.00. 
Further a distinguished quotient $G / N$ of an exceptional group $G$ cannot be cyclic, for if $G / N=\langle N g\rangle$, for some $g \in G$, then $\mu(G) \geqslant \mu(\langle g\rangle) \geqslant \mu(\langle N g\rangle)=\mu(G / N)$. The following examples show that exceptional groups exist.

Example 0.1. Let $G_{1}=\left\langle x, y \mid x^{8}=y^{4}=1, \quad x^{y}=x^{-1}\right\rangle$ and $N_{1}=\left\langle x^{4} y^{2}\right\rangle \simeq \mathbb{Z}_{2}$, so that $N_{1}$ is contained in the centre $Z\left(G_{1}\right)=\left\langle x^{4}, y^{2}\right\rangle$ of $G_{1}$. Then $G_{1}$ is a group of order 32 and $\mu\left(G_{1}\right)=12$, a minimal representation $\phi: G_{1} \rightarrow S_{12}$ being induced by $x \phi=(1,2,3,4,5,6,7,8)$ and $y \phi=(1,8)(2,7)(3,6)(4,5)(9,10,11,12)$. However $G_{1} / N_{1} \simeq\left\langle x, y \mid x^{8}=1, y^{2}=x^{4}, x^{y}=x^{-1}\right\rangle$ is a generalised quaternion group and by $\left[\boldsymbol{6}\right.$, Theorem 1] its minimal degree is $\left|G_{1} / N_{1}\right|=16$. Thus $G_{1}$ is exceptional with distinguished subgroup $N_{1}$.

Example 0.2. Let $G_{2}=\langle x, y, n| x^{8}=n^{2}=1, \quad y^{2}=x^{4}, \quad x^{y}=x^{-1} n, \quad n^{x}=n^{y}=$ $n\rangle$ and $N_{2}=\langle n\rangle \simeq \mathbb{Z}_{2}$, so $N_{2} \leqslant Z\left(G_{2}\right)=\left\langle n, x^{4}\right\rangle$. Again $\left|G_{2}\right|=32$ and $\mu\left(G_{2}\right)=12$, a minimal representation $\psi: G_{2} \rightarrow S_{12}$ being induced by $x \psi=(1,2,3,4,5,6,7,8)(9,10)$, $y \psi^{\prime}=(1,2,5,6)(3,4,7,8)(9,11)(10,12)$ and $n \psi=(1,5)(2,6)(3,7)(4,8)(9,10)(11,12)$. As before $G_{2} / N_{2}$ is generalised quaternion so $G_{2}$ is exceptional with distinguished subgroup $N_{2}$.

In Section 1 we show that exceptional groups have order at least 32, and show that the only exceptional groups of that order are those of the previous examples. We explore properties of minimal exceptional groups: an exceptional group is called $S$-minimal, $Q$-minimal or $S Q$-minimal if no proper subgroup, quotient or section respectively is exceptional. For example, we show a distinguished subgroup $N$ of an SQ-minimal exceptional group $G$ is contained in the Frattini subgroup $\Phi(G)$ and may be taken to be elementary abelian.

In Section 1 we use the well-known fact that if $G$ is a transitive imprimitive permutation group, with $\bar{G}$ the permutation group induced on the blocks of imprimitivity, and if $G_{B}^{B}$ denotes the action of the set stabiliser $G_{B}$ restricted to some block $B$, then $G$ can be embedded in the wreath product $G_{B}^{B}$ wr $\bar{G}$.

In Section 2 we give several families of examples of exceptional groups together with two general constructions. All our nontrivial examples have distinguished quotients with cyclic centres. As remarked earlier a distinguished quotient cannot be cyclic, but the authors do not know whether it can be abelian. That it cannot be elementary abelian is proved in [8] where also a characterisation is given of groups $G$ with an elementary abelian quotient $G / N$ such that $\mu(G)=\mu(G / N)$. In Section 3 we show that $\mu\left(T_{1} \times \ldots \times T_{r}\right)=\mu\left(T_{1}\right)+\ldots+\mu\left(T_{r}\right)$ for simple groups $T_{1}, \ldots, T_{r}$, which is a contribution to the investigations of Johnson [6] and Wright [12] of the minimal degree of direct products of groups. It is always true that $\mu(G \times H) \leqslant \mu(G)+\mu(H)$, but it is an open problem to characterise when equality occurs. For example equality occurs if 
$G$ and $H$ are nilpotent and non-trivial [12, Corollary 2].

Throughout $D_{2 r}=\left\langle a, b \mid a^{r}=b^{2}=1, \quad a^{b}=a^{-1}\right\rangle$ and $Q_{2^{n}}=\langle a, b| a^{2^{n-1}}=$ 1, $\left.\quad a^{2^{n-2}}=b^{2}, \quad a^{b}=a^{-1}\right\rangle$ denote the dihedral group of order $2 r$ and the generalised quaternion group of order $2^{n}$ respectively.

\section{Minimal EXCEPTIONAL GROUPS}

Throughout this Section, $G$ will be an exceptional group with distinguished subgroup $N$.

\section{LEMMA 1.1.}

(a) If $G$ is $S$-minimal then $N$ is contained in the Frattini subgroup $\Phi(G)$, that is $N$ is contained in each maximal subgroup of $G$.

(b) If $M$ is a normal subgroup of $G$ contained in $N$ then either $M$ is a distinguished subgroup of $G$ or $G / M$ is exceptional with distinguished subgroup $N / M$. In particular if $G$ is $Q$-minimal then every nontrivial normal subgroup of $G$ contained in $N$ is distinguished.

\section{PROOF:}

(a) Suppose that $M$ is a maximal subgroup of $G$ which does not contain $N$. Then $G=N M$, so $G / N \simeq M /(M \cap N)$ and, since $M$ is not exceptional, $\mu(G / N)=\mu(M /(M \cap N)) \leqslant \mu(M) \leqslant \mu(G)$, which contradicts the fact that $N$ is distinguished. Thus $N$ is contained in every maximal subgroup of $G$.

(b) Let $M$ be a normal subgroup of $G$ contained in $N$. If $M$ is not distinguished then $\mu(G / M) \leqslant \mu(G)<\mu(G / N)=\mu((G / M) /(N / M))$.

Thus if $G$ is SQ-minimal we can take $N$ to be a minimal normal subgroup of $G$ contained in the nilpotent group $\Phi(G)$, so we can assume that $N$ is elementary abelian. Next we look at the action of a distinguished subgroup in a minimal representation.

LEMMA 1.2. Suppose that an exceptional group $G$ is minimally represented on $X=\bigcup_{i \leqslant r} X_{i}$ with orbits $X_{1}, \ldots, X_{r}$. Then a distinguished subgroup $N$ is intransitive on each $X_{i}$ if $G$ is $S$-minimal, and is nontrivial on each $X_{i}$ if $G$ is Q-minimal. In particular if $G$ is $S Q$-minimal then $G$ acts imprimitively on each $X_{i}$ so $\left|X_{i}\right|$ is a number greater than 1 which is not prime.

Proof: If $G$ is S-minimal then $N \leqslant \Phi(G)$ by Lemma 1.1. If further $N$ were transitive on some $X_{i}$ then $G=N G_{X}$ for $x \in X_{i}$, so $G=G_{x}$ by [5, III 3.2]. In that case $\left|X_{i}\right|=1$, contradicting the minimality of $|X|$.

Suppose now that $G$ is Q-minimal and $N$ fixes some $X_{i}$ pointwise; put $Y=$ $X \backslash X_{i}$. Then $G$ is a subdirect product of $G^{X_{i}}$ and $G^{Y}$. Since $N^{X_{i}} \simeq 1$ we have 
$N \simeq N^{Y}$ and it follows that $G / N<G^{X_{i}} \times\left(G^{Y} / N^{Y}\right)$. Also $G^{Y}$ is not exceptional since $G$ is Q-minimal. Hence $\mu(G / N) \leqslant \mu\left(G^{X_{i}} \times\left(G^{Y} / N^{Y}\right)\right) \leqslant \mu\left(G^{X_{i}}\right)+\mu\left(G^{Y} / N^{Y}\right) \leqslant$ $\mu\left(G^{X_{i}}\right)+\mu\left(G^{Y}\right) \leqslant\left|X_{i}\right|+|Y|=\mu(G)$, contradicting the fact that $N$ is distinguished. Thus $N$ is nontrivial on each $X_{i}$.

Thus if $G$ is SQ-minimal then the $N$-orbits in $X_{i}$, for each $i$, are nontrivial blocks of imprimivity for $G^{X_{i}}$, so in particular $\left|X_{i}\right|>1$ and cannot be prime.

Proposition 1.3. Supppose that $H$ is a finite group with a nontrivial abelian normal subgroup $K$. Then $H$ has an abelian normal subgroup $L$ containing $K$ for which $\mu(H / L)<\mu(H)$ and the prime divisors of $|L|$ and $|K|$ are the same. If $K$ is elementary abelian then $L$ may be chosen to be elementary abelian.

Proof: Let $H$ be minimally represented on a set $X$ with orbits $X_{1}, \ldots, X_{r}$. For each $i$ let $Y_{i}$ be the set of $K$-orbits in $X_{i}$, so the action of $H^{X_{i}}$ induces a group $H^{(i)}$ of permutations of $Y_{i}$. For each $i$ choose some $B_{i} \in Y_{i}$. Put $H_{i}=H_{B_{i}}^{B_{i}}$, the restriction to $B_{i}$ of the set-stabiliser of $B_{i}$, and $H_{i}^{*}=H_{i}$ wr $H^{(i)}$. Then $H_{i} \geqslant K^{-B_{i}}$ and $H^{X_{i}}<H_{i}^{*}$. Since $K$ is abelian, its action on each $B_{i}$ is regular and $K^{B_{i}}$ is self-centralising in $H_{i}$, by $[11,4.4]$. Hence $\left|K^{B_{i}}\right|=\left|B_{i}\right|, H_{i} / K^{B_{i}} \lesssim$ Aut $\left(K^{B_{i}}\right)$ and $\left|X_{i}\right|=\left|K^{B_{i}}\right|\left|Y_{i}\right|$. Now let $L_{i}$ be the subgroup of $H_{i}^{*}$ generated by the conjugates of $K^{B_{i}}$ by elements of $H$, that is, $L_{i}$ is the direct product of $\left|Y_{i}\right|$ copies of $K^{B_{i}}$, so $K^{X_{i}} \lesssim L_{i} \triangleleft H_{i}^{*}$. Put $L^{*}=\prod_{i} L_{i}$ and $H^{*}=\prod_{i} H_{i}^{*}$, so $L^{*} \triangleleft H^{*}$ and $H \lesssim H^{*}$. Now put $L=H \cap L^{*}$ (after identifying $H$ with its image under the embedding), so $L$ is abelian and $K \leqslant L$. Then $\mu(H / L)=\mu\left(H L^{*} / L^{*}\right) \leqslant \mu\left(H^{*} / L^{*}\right)=\mu\left(\prod_{i}\left(H_{i} / K^{B_{i}}\right)\right.$ wr $\left.H^{(i)}\right) \leqslant$ $\mu\left(\prod_{i}\left(\right.\right.$ Aut $\left.K^{B_{i}}\right)$ wr $\left.H^{(i)}\right)$. Since $K$ is nontrivial, at least one $K^{B_{i}}$ is nontrivial, in which case $\mu\left(\left(\right.\right.$ Aut $\left.K^{B_{i}}\right)$ wr $\left.H^{(i)}\right) \leqslant\left(\left|K^{B_{i}}\right|-1\right)\left|Y_{i}\right|<\left|K^{B_{i}}\right|\left|Y_{i}\right|=\left|X_{i}\right|$. Thus $\mu(H / L)<\sum_{i}\left|X_{i}\right|=|X|=\mu(H)$. Observe that $|L|$ and $|K|$ have the same prime divisors and if $K$ is elementary abelian then so is $L$.

We have immediately from Lemma 1.1 and Proposition 1.3 the following:

LEMMA 1.4. If $G$ is an $S Q$-minimal exceptional group then $G$ has elementary abelian normal subgroups $N$ and $M$ for which $N$ is distinguished, $M$ is not distinguished and $M$ properly contains $N$.

We now show that groups $G_{1}$ and $G_{2}$ in the introduction are the smallest exceptional groups.

THEOREM 1.5. There are no exceptional groups of order less than 32. If $G$ is exceptional of order 32 then $G$ is isomorphic to $\left\langle x, y \mid x^{8}=y^{4}=1, x^{y}=x^{-1}\right\rangle$ or 
$\left\langle x, y, n \mid x^{8}=n^{2}=1, y^{2}=x^{4}, x^{y}=x^{-1} n, n^{x}=n^{y}=n\right\rangle$.

Proof: It is sufficient to prove the theorem for SQ-minimal exceptional groups. So let $G$ be SQ-minimal of order $\leqslant 32$. By Lemma 1.4, $G$ has a distinguished subgroup $N$ which is elementary abelian and properly contained in an elementary abelian normal subgroup $M$. Note that $G \neq M$ since $G$ must be non-abelain.

We claim first that $\mu(G)>8$. If $\mu(G) \leqslant 8$, then by Lemma 1.2 a minimal representation of $G$ on a set $X$ is transitive of degree 4,6 or 8 or splits up into two transitive constituents of degree 4. If $\mu(G)=4$ then $G<S_{4}$ and $|G / N| \geqslant \mu(G / N)>\mu(G)=4$, from which it follows easily that $G=S_{4}$ and $N=\mathbb{Z}_{2} \times \mathbb{Z}_{2}$, so $\mu(G / N)=\mu\left(S_{3}\right)=3$, a contradiction. If $\mu(G)=6$ then $G$ is a transitive subgroup of $S_{6}$ such that the $N$-orbits are nontrivial blocks of imprimivity of $G$, and since $N<M<G$ it follows that $|N|=2,3$, or 4 . In this case let $G^{*}$ be the normaliser of $N$ in $S_{6}$, so $G \leqslant G^{*}$. If $|N|=2$ then $G^{*} \simeq \mathbb{Z}_{2}$ wr $S_{3}$ and $G^{*} / N \simeq S_{4}$, so $\mu(G / N) \leqslant 4$. If $|N|=3$ then $G^{*} \simeq \mathbb{Z}_{3}^{2} \rtimes \mathbb{Z}_{2}^{2}$ and $\mu(G / N) \leqslant \mu\left(D_{12}\right)=5$. If $|N|=4$ then $G^{*} \simeq \mathbb{Z}_{2}$ wr $S_{3}$ and $G^{*} / N \simeq \mathbb{Z}_{2} \times S_{3}$, so $\mu(G / N) \leqslant 5$. Each of these contradicts the fact that $\mu(G / N)>6$. Finally suppose $\mu(G)=8$, so either $G$ is a transitive subgroup of $S_{8}$ or a subgroup of $S_{4} \times S_{4}$ with two orbits of size 4 . If $|N|>2$ then $|N| \geqslant 4$ so $|G|=|G / N||N| \geqslant \mu(G / N)|N|>\mu(G)|N| \geqslant 32$ which contradicts the fact that $|G| \leqslant 32$. Hence $|N|=2$. Let $G^{*}$ be the normaliser of $N$ in $S_{8}$, so $G^{*}=\mathbb{Z}_{2}$ wr $S_{4}$ and $G^{*} / N$ is isomorphic to the following permutation group of degree $8,\langle(12)(34)(56)(78),(13)(24)(57)(68),(15)(48)(26)(37)$, $(25)(17)(46)(38),(28)(14)(67)(35),(358)(467),(12)(45)(78)(36)\rangle$, which shows that $\mu(G / N) \leqslant 8$, a contradiction. This completes the proof that $\mu(G)>8$.

Thus $10 \leqslant \mu(G / N) \leqslant|G / N| \leqslant 16$. Since $G / N$ is not cyclic $|G / N| \neq 11,13$ or 15. If $|G / N|=10$ then $\mu(G / N)=\mu\left(D_{10}\right)=5$; if $|G / N|=12$ then $G / N$ is one of $\mathbf{Z}_{3} \times \mathbb{Z}_{2}^{2}, D_{12}, Z_{3} \rtimes \mathbf{Z}_{4}$ or $A_{4}$, so $\mu(G / N) \leqslant 7$; if $|G / N|=14$ then $G / N \simeq D_{14}$ so. $\mu(G / N)=7$. Each of these leads to a contradiction. Hence $|G / N|=16$ and $|N|=2$, and there are no exceptional groups of order less than 32.

Since $|G|=32$, by Lemma 1.2 we have that $\mu(G)$ is a multiple of 4 , so $\mu(G) \geqslant 12$, and hence $\mu(G / N)>12$. There are 13 non-cyclic groups of order 16 (see $[10]$ ). The only group on this list with minimal degree more than 12 is $Q_{16}$. Indeed $\mu\left(Q_{16}\right)=16$. Thus $G$ is an extension of $N=\langle n\rangle \simeq \mathbf{Z}_{2}$ by $Q_{16}$, which is not a direct product and does not contain an element of order 16 or subgroup isomorphic to $Q_{16}$. There are only the following possibilities for $G$ :

$$
\begin{aligned}
& G_{1}=\left\langle x, y, n \mid x^{8}=n^{2}=1, \quad y^{2}=x^{4} n, \quad x^{y}=x^{-1}, \quad n^{x}=n^{y}=n\right\rangle \\
& G_{2}=\left\langle x, y, n \mid x^{8}=n^{2}=1, \quad y^{2}=x^{4}, \quad x^{y}=x^{-1} n, \quad n^{x}=n^{y}=n\right\rangle ; \\
& G_{3}=\left\langle x, y, n \mid x^{8}=n^{2}=1, \quad y^{2}=x^{4} n, \quad x^{y}=x^{-1} n, \quad n^{x}=n^{y}=n\right\rangle .
\end{aligned}
$$


But $G_{1}$ is the exceptional group of Example 0.1 ; to see this observe that $n=x^{4} y^{2}$ so $y^{4}=1$. Further $G_{2}$ is the exceptional group of Example 0.2 and $G_{3} \simeq G_{2}$; to see this observe, for $x, y, n \in G_{3}$, that $(y x)^{2}=x^{4}$ and $x^{y x}=x^{-1} n$. This completes the proof of the theorem.

We complete this section by proving:

Proposition 1.6. Any $S$-minimal exceptional group with nilpotent distinguished quotient is a $p$-group for some prime $p$.

Proof: Let $G$ be S-minimal, so $N \leqslant \Phi(G)$ by Lemma 1.1. If $G / N$ is nilpotent then $G$ is also nilpotent by [5, III. 3.5]. Let $p$ be a prime divisor of $|G|$. If $G$ is not a $p$-group then $G=P \times Q$ where $P$ and $Q$ are nontrivial and have coprime order. As $G$ is $\mathrm{S}$-minimal neither $P$ nor $Q$ is exceptional, and as $N$ is nilpotent $N=(N \cap P) \times(N \cap Q)$. Thus $\mu(G / N)=\mu(P /(N \cap P) \times Q /(N \cap Q)) \leqslant$ $\mu(P /(N \cap P))+\mu(Q /(N \cap Q)) \leqslant \mu(P)+\mu(Q)=\mu(G)$, by [6, Proposition 2], contradicting the inequality $\mu(G / N)>\mu(G)$. Thus $G$ is a $p$-group.

Also the smallest example $G$, if it exists, of an exceptional group with abelian distinguished quotient $G / N$ must be a $p$-group; for by the proof of Lemma 1.1(a) $N$ is contained in $\Phi(G)$, and as in the proof of Proposition 1.6, $G$ is a $p$-group.

\section{EXAMPLES OF EXCEPTIONAL GROUPS}

In this Section we give examples of classes of exceptional groups. We begin with a general class of examples of exceptional $p$-groups.

TheOREM 2.1. Let $p$ be a prime and let $G_{1}, G_{2}$ be non-cyclic $p$-groups with cyclic centres $Z_{1}=\left\langle a_{1}\right\rangle$ and $Z_{2}=\left\langle a_{2}\right\rangle$ respectively of the same order.

(a) If $p$ is odd then $G_{1} \times G_{2}$ is an exceptional group with distinguished subgroup $N=\left\langle\left(a_{1}, a_{2}\right)\right\rangle$.

(b) If $p=2, \mu\left(G_{1}\right)>\mu\left(G_{2}\right)$ and $G_{2}$ is not a generalised quaternion group, then $G_{1} \times G_{2}$ is exceptional with distinguished subgroup $N=\left\langle\left(a_{1}, a_{2}\right)\right\rangle$.

Proof: First we claim that $H=\left(G_{1} \times G_{2}\right) / N$ has cyclic centre $\left(Z_{1} \times Z_{2}\right) / N=$ $\left\langle\left(a_{1}, 1\right) N\right\rangle=\left\langle\left(1, a_{2}\right) N\right\rangle$. This is true since $(u, v) N \in Z(H)$ if and only if, for all $(g, h) \in G_{1} \times G_{2}$, we have $[(u, v),(g, h)]=([u, g],[v, h]) \in N$, that is for all $(g, h) \in$ $G_{1} \times G_{2}$ there is some integer $i$ for which $[u, g]=a_{1}^{i}$ and $[v, h]=a_{2}^{i}$. Taking $h=1$ this condition becomes $[u, g]=1$ for all $g \in G_{1}$, that is $u \in Z_{1}$, while taking $g=1$ yields $v \in Z_{2}$.

Thus $G_{1}, G_{2}$ and $H$ have cyclic centres and so by Johnson [6, Theorem 3] all minimal representations of $G_{1}, G_{2}$ and $H$ are transitive so that $\mu\left(G_{1}\right)=p^{n_{1}}, \mu\left(G_{2}\right)=$ $p^{n_{2}}$ and $\mu(H)=p^{n}$ for some $n_{1}, n_{2}$ and $n$. 
We may assume that $\mu\left(G_{1}\right) \geqslant \mu\left(G_{2}\right)$. Since $G_{2}$ is neither cyclic nor (if $p=2$ ) generalised quaternion, $G_{2}$ has a subgroup $B=\langle b\rangle \neq 1$ such that $B \cap Z_{2}=1$ (see Gorenstein $[4,5.4 .10])$. Then $G_{1} \times B \simeq\left(G_{1} \times B\right) N / N \leqslant H$ so that $\mu(H) \geqslant \mu\left(G_{1} \times B\right)$ and $\mu\left(G_{1} \times B\right)=\mu\left(G_{1}\right)+\mu(B)$ by Wright [12, Theorem 2]. Thus $\mu(H)>\mu\left(G_{1}\right)$, that is, $n>n_{1}$ so that $\mu(H) \geqslant p \mu\left(G_{1}\right)$. For $p$ odd we have $\mu(H)>2 \mu\left(G_{1}\right) \geqslant$ $\mu\left(G_{1}\right)+\mu\left(G_{2}\right)=\mu\left(G_{1} \times G_{2}\right)$, proving part (a). For $p=2$, if $\mu\left(G_{1}\right)>\mu\left(G_{2}\right)$ we again have $\mu(H)>\mu\left(G_{1}\right)+\mu\left(G_{2}\right)=\mu\left(G_{1} \times G_{2}\right)$, proving part (b).

Remarks 2.2. The theorem shows that the following classes of 2-groups are exceptional:

(a) $D_{2^{n}} \times D_{2^{m}}$ for $n>m \geqslant 3$, with distinguished quotient the central product $D_{2^{n}} * D_{2^{m}}$, and

(b) $Q_{2^{n}} \times D_{2^{m}}$ for $n \geqslant m \geqslant 3$, with distinguished quotient $Q_{2^{n}} * D_{2^{m}}$.

The smallest of these examples is $Q_{8} \times D_{8}$ of order 64 (which is larger than the examples given in the introduction). By repeated applications of the theorem we can obtain other classes of exceptional 2-groups, for example:

(c) $\left(D_{16} *\left(* D_{8}\right)^{n}\right) \times D_{8}$ with distinguished quotient $D_{16} *\left(* D_{8}\right)^{n+1}$, where $(* K)^{n}$ denotes the $n$-fold central product $K * \ldots * K$.

(d) $\left(Q_{8} *\left(* D_{8}\right)^{n}\right) \times D_{8}$ with distinguished quotient $Q_{8} *\left(* D_{8}\right)^{n+1}$

In fact the groups $D_{2^{n}} \times D_{2^{m}}, Q_{2^{n}} \times D_{2^{m}}$, and $Q_{2^{n}} \times Q_{2^{m}}$ are exceptional for all values of $n$ and $m$ at least 3 , but this does not follow from Theorem 2.1. First we make the following observation:

LEMMA 2.3. Suppose that $G$ is an abelian group generated by $x$ and $y$, and that $H$ is a subgroup of $G$ for which $H \cap\langle x\rangle=H \cap\langle y\rangle=1$. Then $H=\left\langle x^{i} y^{i}\right\rangle$ for some $i$, $j$ for which $\left|x^{i}\right|=\left|y^{j}\right|$.

Proof: If $H=1$ then the statement of the lemma holds. Suppose $H \neq 1$, and let $i$ be the least positive integer such that $x^{i} y^{j} \in H$ for some $j$. If also $x^{k} y^{l} \in G$ then $x^{k}=x^{i \alpha}$ for some $\alpha$, so $x^{k} y^{l}\left(x^{i} y^{j}\right)^{-\alpha}=y^{l-j \alpha} \in H$. Thus $y^{l-j \alpha}=1$, so $y^{l}=y^{j \alpha}$ and $x^{k} y^{l} \in\left\langle x^{i} y^{j}\right\rangle$. This shows that $H=\left\langle x^{i} y^{j}\right\rangle$. If $\left|x^{i}\right| \neq\left|y^{j}\right|$ then some nontrivial power of $x^{i} y^{j}$ lies in $\langle x\rangle$ or $\langle y\rangle$, which is impossible. Hence, $\left|x^{i}\right|=\left|y^{j}\right|$.

Proposition 2.4. Suppose that $n$ and $m$ are integers greater than 2. Then $D_{2^{n}} \times D_{2^{m}}, Q_{2^{n}} \times D_{2^{m}}$, and $Q_{2^{n}} \times Q_{2^{m}}$ are all exceptional groups with distinguished quotients $D_{2^{n}} * D_{2^{m}}, Q_{2^{n}} * D_{2^{m}}$ and $Q_{2^{n}} * Q_{2^{m}}$ respectively. Further the direct products have minimal degrees $2^{n-1}+2^{m-1}, 2^{n}+2^{m-1}$ and $2^{n}+2^{m}$ respectively, 
while the central products have minimal degrees as follows:

$$
\begin{array}{ll}
\mu\left(D_{2^{n}} * D_{2^{m}}\right)=2^{m} & \text { if } n \leqslant m ; \\
\mu\left(Q_{2^{n}} * Q_{2^{m}}\right)=2^{m} & \text { if } n \leqslant m ;
\end{array} \begin{array}{ll}
2^{m+1} & \text { if } n \leqslant m, \\
2^{n+1} & \text { if } n \geqslant m .
\end{array}
$$

Proof: The minimal degrees of the direct products follow from Wright $[12$, Theorem 2] and from the facts that $\mu\left(D_{2^{m}}\right)=2^{m-1}$ and $\mu\left(Q_{2^{n}}\right)=2^{n}$ (see Wright [12, pp.901-902] and Johnson [6, Theorem 1]). We need to determine the minimal degrees of the central products.

First suppose that $n \leqslant m$ and embed $D_{2^{n}}$ naturally in $D_{2^{m}}$. Define

$$
\phi: D_{2^{n}} \times D_{2^{m}} \rightarrow \operatorname{Sym}\left(D_{2^{m}}\right)
$$

by $(g, h) \phi: x \mapsto g^{-1} x h$ for all $x \in D_{2^{m}}$. Then $\phi$ is a permutation representation with kernel $\left\{(g, g) \mid g \in Z\left(D_{2^{m}}\right)\right\}$ and hence $D_{2^{n}} * D_{2^{m}} \simeq\left(D_{2^{n}} \times D_{2^{m}}\right) / \operatorname{Ker} \phi<$ $\operatorname{Sym}\left(D_{2^{m}}\right)$. Thus $\mu\left(D_{2^{n}} * D_{2^{m}}\right) \leqslant 2^{m}$. On the other hand $\mathbb{Z}_{2} \times D_{2^{m}}<D_{2^{n}} * D_{2^{m}}$ and so $2^{m-1}<\mu\left(\mathbb{Z}_{2} \times D_{2^{m}}\right) \leqslant \mu\left(D_{2^{n}} * D_{2^{m}}\right)$; by Johnson [6, Theorem 3] and since $D_{2^{n}} * D_{2^{n}}$ has cyclic centre, $D_{2^{n}} * D_{2^{m}}$ has a transitive minimal representation so that $\mu\left(D_{2^{n}} * D_{2^{m}}\right)$ is a power of 2 . It follows that $\mu\left(D_{2^{n}} * D_{2^{m}}\right)=2^{m}$. If $n \leqslant m$ a similar argument shows that $\mu\left(Q_{2^{n}} * Q_{2^{m}}\right)=2^{m}$ (noting that $Q_{2^{m}} \lesssim Q_{2^{n}} * Q_{2^{m}}$ gives $\left.2^{m}=\mu\left(Q_{2^{m}}\right) \leqslant \mu\left(Q_{2^{n}} * Q_{2^{m}}\right)\right)$.

For the remaining case we shall denote $H=Q_{2^{n}} * D_{2^{m}}$ by $H=\langle a, b, c, d| a^{2^{n-1}}=$ $c^{2^{m-1}}=d^{2}=1, \quad a^{2^{n-2}}=b^{2}=c^{2^{m-2}}, \quad b a=a^{-1} b, \quad d c=c^{-1} d, \quad[a, c]=[a, d]=$ $[b, c]=[b, d]=1\rangle$. Now $Q_{2^{n}} \times \mathbb{Z}_{2}<H$ so that $\mu(H) \geqslant 2^{n}+2>2^{n}$. Further $\left\langle b^{2}\right\rangle \simeq \mathbb{Z}_{2}$ is the unique minimal normal subgroup of $H$ since $\left[a^{i} b^{j} c^{k} d^{l}, b\right]=a^{ \pm 2 i}$ and $\left[a^{i} b^{j} c^{k} d^{l}, d\right]=c^{ \pm 2 k}$. It follows that a minimal faithful representation for $H$ must be transitive (by Johnson [6, Theorem 3] for $Z(H)$ must be cyclic). Thus $\mu(H)$ is a power of 2 and hence $\mu(H) \geqslant 2^{n+1}$. Also $\mu(H)=|H: K|$ where $K$ is a subgroup maximal such that $b^{2} \notin K$. Taking $K \geqslant\left\langle a c^{2^{m-n}}\right\rangle \simeq \mathbb{Z}_{2^{n-2}}$ if $m \geqslant n$ or $K \geqslant\left\langle a^{2^{n-m}} c\right\rangle \simeq \mathbb{Z}_{2^{m-2}}$ if $m \leqslant n$, we obtain $\mu(H) \leqslant 2^{m+1}$ and $\mu(H) \leqslant 2^{n+1}$ respectively. If $m \leqslant n$ we conclude that $\mu(H)=2^{n+1}$.

So suppose that $m>n$. Since $b^{2} \notin K$ we have that $K \cap\langle a\rangle=K \cap\langle c\rangle=1$. Hence $K \cap\langle a, c\rangle=\left\langle a^{i} c^{j}\right\rangle$ for some $i, j$ by Lemma 2.3. If $K \leqslant\langle a, c\rangle$, then, since the largest cyclic subgroups not containing $b^{2}$ have order $2^{n-2}$, it follows that $|K|=2^{n-2}$ and $\mu(H)=2^{m+1}$. So suppose that $K$ contains an element of $H \backslash\langle a, c\rangle$, say $y=b^{\delta} d^{\varepsilon} a^{k} c^{l}$ where $\delta$ and $\varepsilon$ are 0 or 1 and $\delta+\varepsilon \geqslant 1$. Then $K \cap\langle a, c\rangle$ contains $\left(a^{i} c^{j}\right)^{y}=a^{i^{\prime}} c^{j^{\prime}}$ 
where $i^{\prime}=(-1)^{\delta} i, j^{\prime}=(-1)^{\epsilon} j$; since $K \cap\langle a, c\rangle=\left\langle a^{i} c^{j}\right\rangle$ it follows that $\delta=\varepsilon=1$ and $K$ contains $\left(b d a^{k} c^{l}\right)^{2}=b^{2}$ which is a contradiction.

All our examples so far in this Section of exceptional 2-groups have been direct products. Our final class of examples of exceptional 2-groups is not a class of direct products and its smallest member, $H_{2,3}$ is Example 0.1, of order 32, given in the Introduction.

Proposition 2.5. The groups

$$
H_{m, n}=\left\langle x, y \mid x^{2^{n}}=y^{2^{m}}=1, \quad x^{y}=x^{-1}\right\rangle
$$

are exceptional of order $2^{m+n}$ if $2 \leqslant m<n$.

Proof: Put $N=\left\langle x^{2^{n-1}} y^{2}\right\rangle \triangleleft H_{m, n}$. Then $H_{m, n} / N$ is genaralised quaternion of order $2^{n+1}$, so has minimal degree $2^{n+1}$. On the other hand, $\mu\left(H_{m, n}\right) \leqslant 2^{m}+2^{n}$ since

$$
\begin{aligned}
H_{m, n} \simeq & \left\langle\left(1,2, \ldots, 2^{n}\right),\left(1,2^{n}\right)\left(2,2^{n}-1\right) \ldots .\right. \\
& \left.\left(2^{n-1}, 2^{n-1}+1\right)\left(2^{n}+1,2^{n}+2, \ldots, 2^{n}+2^{m}\right)\right\rangle .
\end{aligned}
$$

This shows that $H_{m, n}$ is exceptional if $2 \leqslant m<n$.

We now turn to exceptional $p$-groups where $p$ is odd. It follows from Theorem 2.1 that if $G=\mathbb{Z}_{p}$ wr $\mathbb{Z}_{p}$ then $G \times G$ is exceptional of order $p^{2(p+1)}$. (In fact a minimal representation for the central product $G * G$ is induced by $\phi: G \times G \rightarrow \operatorname{Sym} G$ where $(g, h) \phi: x \mapsto g^{-1} x h$.) A smaller class of groups is given by $G \times G$ of order $p^{6}$ where $G$ is an extraspecial group of order $p^{3}$, that is, $G^{\prime}=Z(G)=\Phi(G) \simeq \mathbb{Z}_{p}$. (Such groups are described in $[4,5.5 .1])$. The authors do not know if there are any exceptional $p$-groups of order less than $p^{6}$, for $p$ odd.

We now describe a class of exceptional $p$-groups, for $p$ odd, which are not the direct product of groups with cyclic centres.

Proposition 2.6. For $p$ an odd prime and $n$ an integer $\geqslant 1$, define

$$
\begin{aligned}
& G=\mathbb{Z}_{p^{n}}^{p^{n}} \rtimes \mathbb{Z}_{p^{n}}^{p^{n}} \rtimes \mathbb{Z}_{p^{n}} \\
& \quad=\left\langle x_{1}, \ldots, x_{p^{n}}, \quad y_{1}, \ldots, y_{p^{n}}, \quad z\right| x_{i}^{p^{n}}=y_{i}^{p^{n}}=z^{p^{n}}=1 \quad \text { for all } i \\
& {\left[x_{i}, x_{j}\right]=\left[x_{i}, z\right]=\left[y_{i}, y_{j}\right]=1 \quad \text { for all } i, j} \\
& x_{i}^{y_{j}}=x_{i+1} \quad \text { for all } i<p^{n} \text { and all } j, \\
& \left.x_{p^{n}}^{y_{j}}=x_{1} \text { for all } j, \quad y_{i}^{z}=y_{i+1} \text { for } i<p^{n}, \quad y_{p^{n}}^{z}=y_{1}\right\rangle
\end{aligned}
$$

Then $G$ is an exceptional group of order $p^{n\left(2 p^{n}+1\right)}$ with distinguished subgroup $N=$ $\left\langle x_{1} \cdots x_{p^{n}} y_{1}^{-1} \cdots y_{p^{n}}^{-1}\right\rangle$. 
Proof: First $\mu(G) \leqslant 2 p^{2 n}$ since we have a monomorphism $\phi: G \rightarrow S_{2 p^{2 n}}$ defined by

$$
\begin{aligned}
& x_{i} \phi=\left(i p^{n}-p^{n}+1, i p^{n}-p^{n}+2, \ldots, i p^{n}\right) \quad \text { for } i=1, \ldots, p^{n} \\
& y_{i} \phi=b \cdot\left(p^{2^{n}}+i p^{n}-p^{n}+1, p^{2 n}+i p^{n}-p^{n}+2, \ldots, p^{2 n}+i p^{n}\right)
\end{aligned}
$$

for $i=1, \ldots, p^{n}$ where

$$
b=\prod_{1 \leqslant i \leqslant p^{n}}\left(i, p^{n}+i, 2 p^{n}+i, \ldots,\left(p^{n}-1\right) p^{n}+i\right)
$$

and

$$
z \phi=\prod_{1 \leqslant i \leqslant p^{n}}\left(p^{2 n}+i, p^{2 n}+p^{n}+i, \ldots, r^{2 n}+\left(p^{n}-1\right) p^{n}+i\right)
$$

Moreover since $G / N \geqslant\left\langle x_{1} N\right\rangle \times \ldots \times\left\langle x_{p^{n}} N\right\rangle \times\langle z N\rangle \simeq \mathbb{Z}_{p^{n}}^{p^{n}+1}$ we have $\mu(G / N) \geqslant$ $p^{2 n}+p^{n}$. Also $G / N$ has centre $\left\langle x_{1} \ldots x_{p^{n}} N\right\rangle \simeq \mathbb{Z}_{p^{n}}$, so by $[\mathbf{6}$, Theorem 3] $\mu(G / N)$ is a power of $p$ and hence $\mu(G / N) \geqslant p^{2 n+1}>\mu(G)$, which proves that $G$ is exceptional.

Next we look for exceptional groups which are not $p$-groups. We find first of all a trivial construction.

Proposition 2.7. If $G$ and $H$ are groups and $G$ is an exceptional group then $G \times H$ is also an exceptional group if either

(a) $(|G|,|H|)=1$, or

(b) $G$ and $H$ are both nilpotent.

Proof: The result is clearly true if $H$ is trivial, so suppose $H$ is non-trivial. Let $N \triangleleft G$ be a distinguished subgroup for $G$. Then since $(G \times H) /(N \times 1) \simeq(G / N) \times H$ we have $\mu((G \times H) /(N \times 1))=\mu((G / N) \times H)=\mu(G / N)+\mu(H)>\mu(G)+\mu(H)=$ $\mu(G \times H)$ by Johnson [6, Proposition 2] and Wright [12, Corollary 2].

Less trivial examples are provided by direct products of dihedral groups.

Proposition 2.8. For any integer $k=p_{1}^{\alpha_{1}} \ldots p_{s}^{\alpha_{s}}>1$, where the $p_{i}$ are distinct primes, define $\psi(k)=\sum p_{i}^{\alpha_{i}}$ and set $\psi(1)=0$. Then the following hold:

(a) the dihedral group $D_{2^{n} q}$, where $q$ is odd, has minimal degree

$$
\mu\left(D_{2^{n} q}\right)= \begin{cases}2^{n} & \text { if } q=1,1 \leqslant n \leqslant 2 \\ 2^{n-1} & \text { if } q=1, n>2 \\ \psi(q) & \text { if } q>1, n=1 \\ 2^{n-1}+\psi(q) & \text { if } q>1, n>1\end{cases}
$$


(b) Suppose that $2 \leqslant n \leqslant m, q$ and $r$ are odd, and $2^{n} q>4,2^{m} r>4$. Then

$$
\mu\left(D_{2^{n_{q}}} * D_{2^{m_{r}}}\right)= \begin{cases}2^{m-1}+\psi(q)+\psi(r) & \text { if } n=2 \\ 2^{m}+\psi(q)+\psi(r) & \text { if } n>2\end{cases}
$$

(c) the group $D_{2^{n} q} \times D_{2^{m_{r}}}$, where $m, n, q, r$ are as in (b), is exceptional if and only if $2<n<m$.

Proof: Part (a) is proved in Wright [12, pp.901-902]. Let $H=D_{2^{n} q} * D_{2^{m_{r}}}=$ $\langle a, d, c, d| a^{2^{n-1} q}=b^{2}=d^{2}=1, a^{2^{n-2} q}=c^{2^{m-2} r}, a^{b}=a^{-1}, c^{d}=c^{-1},[a, c]=[a, d]=$ $[b, c]=[b, d]=1\rangle$, where $m, n, q, r$ are as in (b). If $n=2$ then $H \simeq D_{2 q} \times D_{2^{m}}$, so $\mu(H)=\mu\left(D_{2 q}\right)+\mu\left(D_{2^{m} r}\right)=\psi(q)+2^{m-1}+\psi(r)$, using (a) and [12, Proposition 2].

Suppose that $n>2$. Since the map

$$
x \mapsto\left(x\left\langle a^{2^{n-1}}, c^{2^{m-1}}\right\rangle, \quad x\left\langle a^{q}, c, d\right\rangle, \quad x\left\langle a, b, c^{r}\right\rangle\right)
$$

defines a monomorphism $H$ into $\left(D_{2^{n}} * D_{2^{m}}\right) \times D_{2 q} \times D_{2 r}$, we have $\mu(H) \leqslant 2^{m}+$ $\psi(q)+\psi(r)$, by Proposition 2.4. Suppose $H_{1}, \ldots, H_{t}$ are subgroups of $H$ such that $\mu(H)=\sum\left|H: H_{i}\right|$ and $\cap \bar{H}_{i}=1$ where $\bar{H}_{i}=\bigcap_{h \in H} H_{i}^{h}$. for each $i$. We first prove that $\left|H: H_{i_{0}}\right|$ is divisible by $2^{m}$ for some $i_{0}$.

Suppose that, for each $i, 2^{m}$ does not divide $\left|H: H_{i}\right|$. Then since $|H|=$ $2^{n+m-1} q r$, we have $2^{n}$ divides each $\left|H_{i}\right|$. Hence each $H_{i}$ has a subgroup $S_{i}$ of order $2^{n}$. Put $z=a^{2^{n-2} q}=c^{2^{m-2} r}$. We will prove that $z \in S_{i}$. Suppose to the contrary that $z \notin S_{i}$. Put $T_{i}=S_{i} \cap\left\langle a^{q}, c^{r}\right\rangle$. By Lemma 2.3, $T_{i}=\left\langle a^{\alpha_{0} q} c^{\beta_{0} r}\right\rangle$ for some $\alpha_{0}, \beta_{0}$ where $\left|a^{\alpha_{0} q}\right|=\left|c^{\beta_{0} r}\right|$, so $\left|T_{i}\right| \leqslant 2^{n-2}$. Put $U_{i}=\left\{h \in S_{i} \mid h=a^{\alpha} b c^{\beta} d\right.$ for some $\left.\alpha, \beta\right\}$. If $g=a^{\alpha} b c^{\beta} d$ and $h=a^{\gamma} b c^{\delta} d$ are elements of $U_{i}$ then $g h=a^{\alpha-\gamma} c^{\beta-\delta} \in T_{i}$, which shows either $\left|U_{i}\right|=0$ or $\left|U_{i}\right|=\left|T_{i}\right|$. Thus $\left|\left\langle T_{i}, U_{i}\right\rangle\right|=\left|T_{i}\right|+\left|U_{i}\right| \leqslant 2\left|T_{i}\right| \leqslant 2^{n-1}<2^{n}$. Hence $S_{i}$ must contain an element of the form $g=a^{\alpha} b c^{\beta r}$ or $h=a^{\gamma q} c^{\delta} d$ for some $\alpha, \beta, \gamma, \delta$. If $g \in S_{i}$ then $g^{2}=c^{2 \beta r} \in S_{i}$, so $c^{2 \beta r}=1$, that is $c^{\beta r}=z$ and $g \in\langle a, b\rangle$. Put $V_{i}=S_{i} \cap\langle a, b\rangle$. If $a^{\mu} b, a^{\nu} b \in V_{i}$ then $a^{\mu-\nu} \in V_{i}$, so $a^{\mu}=a^{\nu}$, since $z \notin S_{i}$, which slows $V_{i}=\left\langle a^{\gamma_{0}} b\right\rangle \cong \mathbf{Z}_{2}$ for some $\gamma_{0}$. Similarly, if $h \in S_{i}$ then $h \in W_{i}=S_{i} \cap\langle c, d\rangle=\left\langle c^{\delta_{0}} d\right\rangle$ for some $\delta_{0}$. If $\left|T_{i}\right|=1$ then $\left|S_{i}\right| \leqslant\left|\left\langle V_{i}, W_{i}\right\rangle\right| \leqslant 4<2^{n}$, contradicting the fact that $\left|S_{i}\right|=2^{n}$. Thus $\left|T_{i}\right| \neq 1$. If $\left|V_{i}\right| \neq 1$ then $z \in\left\langle a^{2 \alpha_{0} q}=\left[a^{-\alpha_{0} q} c^{-\beta_{0} r}, a^{\gamma_{0}} b\right]\right\rangle \leqslant S_{i}$ (for $a^{2 \alpha_{0} q}=1$ implies $a^{\alpha_{0} q}=z=c^{\beta_{0} r}$ and $\left.T_{i}=1\right)$. Similarly if $\left|W_{i}\right| \neq 1$ then $z \in S_{i}$, both leading to contradictions. This proves indeed that $z \in S_{i}$. But $\langle z\rangle \triangleleft H$ so $\cap \bar{H}_{i} \neq 1$, a contradiction. This shows $2^{m}$ divides $\left|H: H_{i_{0}}\right|$ for some $i_{0}$. 
Put $\left|H: H_{i}\right|=2^{\nu_{i}} q_{i}$ where $q_{i}$ is odd, so

$$
\left|H: H_{i}\right| \geqslant \begin{cases}\psi\left(q_{i}\right) & \text { if } \nu_{i}=0 \\ 2 \psi\left(q_{i}\right) & \text { if } \nu_{i}=1 \\ 2^{\nu_{i}}+2 \psi\left(q_{i}\right) & \text { if } \nu_{i}>1\end{cases}
$$

In particular, since $m>2,\left|H: H_{i_{0}}\right| \geqslant 2^{m}+2 \psi\left(q_{i_{0}}\right)$. Denote all the distinct odd prime divisors of $|H|$ by $p_{1}, \ldots, p_{s}$. Put $x=a^{2^{n-1}}$ and $y=c^{2^{m-1}}$, so $\langle x, y\rangle \simeq$ $\left\langle x_{1}\right\rangle \times \ldots \times\left\langle x_{s}\right\rangle \times\left\langle y_{1}\right\rangle \times \ldots \times\left\langle y_{s}\right\rangle$ where, for each $i, x_{i} \in\langle x\rangle, y_{i} \in\langle y\rangle,\left|x_{i}\right|=p_{i}^{\alpha_{i}}$, $\left|y_{i}\right|=p_{i}^{\beta_{i}}$ for some $\alpha_{i}, \beta_{i}$. Put $I=\left\{i \mid \nu_{i} \geqslant 1\right\}$.

Then

$$
\begin{aligned}
\mu(H)=\sum\left|H: H_{i}\right| & =\sum_{i \in I}\left|H: H_{i}\right|+\sum_{i \notin I}\left|H: H_{i}\right| \\
& \geqslant 2^{m}+2 \sum_{i \in I} \psi\left(q_{i}\right)+\sum_{i \notin I} \psi\left(q_{i}\right)
\end{aligned}
$$

For each $i=1$ to $s$ both $\left\langle x_{i}\right\rangle$ and $\left\langle y_{i}\right\rangle$ are normal subgroups of $H$, so we can find $j_{i}$ and $k_{i}$ such that $H_{j_{i}} \cap\left\langle x_{i}\right\rangle=H_{k_{i}} \cap\left\langle y_{i}\right\rangle=1$. Hence $\left|H: H_{j_{i}}\right|$ is divisible by $p_{i}^{\alpha_{i}}$ and $\left|H: H_{k_{i}}\right|$ by $p_{i}^{\beta_{i}}$. From the previous inequality, in order to prove part (b) it is sufficient to show that either $j_{i} \neq k_{i}$, or that $j_{i}=k_{i}$ and either $p_{i}^{\alpha_{i}+\beta_{i}}$ clivides $\left|H: H_{j_{i}}\right|$ or $j_{i} \in I$. So suppose $j_{i}=k_{i}$ and $p_{i}^{\alpha_{i}+\beta_{i}}$ does not divide $\left|H: H_{j_{i}}\right|$. Put $K=H_{j_{i}} \cap\left\langle x_{i}, y_{i}\right\rangle$, so $K \neq 1$. By Lemma 2.3 we have $K=\left\langle x_{i}^{\alpha} y_{i}^{\beta}\right\rangle$ for some $\alpha, \beta$ where $\left|x_{i}^{\alpha}\right|=\left|y_{i}^{\beta}\right| \neq 1$. If $b \in H_{j_{i}}$ then $1 \neq x_{i}^{2 \alpha}=\left[x_{i}^{-\alpha} y_{i}^{-\beta}, b\right] \in H_{j_{i}}$, which contradicts the fact that $H_{j_{i}} \cap\left\langle x_{i}\right\rangle=1$. Hence $b \notin H_{j_{i}}$ so 2 divides $\left|H: H_{j_{i}}\right|$, which shows $j_{i} \in I$. This completes the proof of part (b).

Part (c) follows immediately, using $\mu\left(D_{2^{n} q} \times D_{2^{m_{r}}}\right)=\mu\left(D_{2^{n_{q}}}\right)+\mu\left(D_{2^{m_{r}}}\right)$ and parts (a) and (b).

\section{Direct products of SIMPle groups.}

If $H, K$ are nontrivial groups then clearly $\mu(H \times K) \leqslant \mu(H)+\mu(K)$. Johnson and Wright considered several cases of the question: when is $\mu(H \times K)=\mu(H)+\mu(K)$ ? The answer is affirmative for example if $H$ and $K$ have coprime orders, or if $H$ and $K$ are nilpotent (see [6, Theorem 2] and [12, Corollary 2]), or more generally if $H$ and $K$ contain nilpotent subgroups $H_{1}$ and $K_{1}$ respectively such that $\mu(H)=\mu\left(H_{1}\right)$ and $\mu\left(K^{-}\right)=\mu\left(K_{1}\right)$ (see [12, remark following Corollary 2]). However there are examples where the answer is negative [12, Section 5]. The following result which has been used in [0] to investigate the inclusion problem for finite primitive permutation groups, gives an affirmative answer if $H$ and $K$ are direct products of simple groups. 
THEOREM 3.1. Let $S_{i}$ be a simple group with minimal degree $\mu_{i}$ for $i=1, \ldots, r$. Then if $G=S_{1} \times \ldots \times S_{r}$ we have $\mu(G)=\sum \mu_{i}$.

Proof: In what follows we use the fact that $\mu(S)<|S| / 2$ for a nonabelian simple group $S$ : this is easily seen by considering the (faithful) representation of $S$ on the cosets of a sulggroup of odd prime order.

There is nothing to prove if $r=1$, so assume inductively that $r \geqslant 2$ and that the result is true for fewer factors. Since $\mu(G) \leqslant \sum \mu_{i}$ we need to prove the reverse inequality, that is $\mu(G) \geqslant \sum \mu_{i}$. Let $G$ be minimally represented on $X$, so $|X|=$ $\mu(G)$. If $G$ is intransitive on $X$ then $X=X_{1} \cup X_{2}$ where each $X_{i}$ is nonempty and fixed setwise by $G$. Let $K_{i}$ be the kernel of the action of $G$ on $X_{i}, i=1,2$. Then $G / K_{1}$ is isomorphic to a product of some of the $S_{i}$ and $K_{1}$ to the product of the other $S_{i}$. By the inductive hypothesis $\mu(G)=\left|X_{1}\right|+\left|X_{2}\right| \geqslant \mu\left(G / K_{1}\right)+\mu\left(K_{1}\right) \geqslant \sum \mu_{i}$.

Suppose then that $G$ is transitive on $X$. If each $S_{i}$ is transitive on $X$ then it follows from [11,4.3 and 4.4] that $G=S_{1} \times S_{2}$ where $S_{1}$ is nonabelian and $|X|=$ $\left|S_{1}\right|=\left|S_{2}\right|$. In this case $\mu(G)=|X|>2 \max \left\{\mu_{1}, \mu_{2}\right\} \geqslant \sum \mu_{i}$, a contradiction. Thus at least one of the $S_{i}$ is intransitive on $X$. Choose $S$ to be one of the $S_{i}$ with the shortest orbits in $X$ and let $Y$ be the set of $S$-orbits in $X, B \in Y$, and $K$ the kernel of $G$ on $Y$. Now $K$ is the direct product of some nonabelian simple factors of $G$ and a subgroup of $Z(G)$. Thus the kernel $L$ of $K$ on $B$, being a normal subgroup of $K$, is also a direct product of nonabelian simple factors of $G$ and a subgroup of $Z(K)$. In particular $L$ is normal in $G$ and so is $\frac{1}{2}$-transitive on $X$. Thus $L=1$, that is $K \simeq K^{B}$. If $S \neq K$, say $K \geqslant S \times S^{\prime}$, where $S^{\prime}$ is another simple factor of $G$, then $K^{B} \geqslant S^{B} \times S^{\prime} B$, and by the minimality of $|B|, S^{\prime}$ is transitive on $B$. Thus, by [11,4.3 and 4.4], $S$ is nonabelian, $K \simeq S \times S^{\prime}$ and $|B|=|S|=\left|S^{\prime}\right|>2 \max \left\{\mu(S), \mu\left(S^{\prime}\right)\right\} \geqslant \mu(S)+\mu\left(S^{\prime \prime}\right)$. So $\mu(G)=|Y||B|>|Y|\left(\mu(S)+\mu\left(S^{\prime}\right)\right) \geqslant|Y|+\mu(S)+\mu\left(S^{\prime}\right) \geqslant \mu(G / K)+\mu(S)+\mu\left(S^{\prime}\right)=$ $\sum \mu_{i}$, by the inductive hypothesis, a contradiction. Thus $S=K$ and $\mu(G)=|Y||B| \geqslant$ $\mu(G / K) \mu(S) \geqslant \mu(G / K)+\mu(S)=\sum \mu_{i}$, by the inductive hypothesis, which completes the proof.

We remark that, in the last stage of the proof, $\mu(G / K) \mu(S)>\mu(G / K)+\mu(S)$, giving a contradiction, unless $G / K \simeq S \simeq \mathbb{Z}_{2}$. Thus the proof shows that, unless $Z_{2} \times Z_{2}$ is a factor of $G$, a minimal representation of $G$ must have the same number of orbits as simple factors.

\section{REFERENCES}

[1] D. Easdown, 'The minimal faithful degree of a fundamental inverse semigroup', Bull. Austral. Math. Soc. 35 (1987), 373-378.

[2] D. Easdown, 'The minimal faithful degree of a semilattice of groups', J. Austral. Math. Soc. (to appear). 
[3] D. Easdown, 'Efficient representations of semigroups', submitted, Proceedings of the International. Symposium on Regular Semigroups and Applications (University of Kerala, India, 1986).

[4] D. Gorenstein, Finite Groups (Harper and Row, New York, Evanston, London, 1968).

[5] B.Huppert, Endliche Gruppen I (Springer-Verlag, Berlin, Heidelberg, New York, 1967).

[6] D.L. Johnson, 'Minimal permutation representations of finite groups', Amer. J. Math. 93 (1971), 857-866.

[7] G.I. Karpilovsky, 'The least degree of a faithful representation of abelian groups', Vestnik Khar'kov Gos. Univ. 53 (1970), 107-115.

[8] L.G. Kovacs and Cheryl E. Praeger, 'Finite permutation groups with large abelian quotients', (submitted).

[0] Cheryl E. Praeger, The inclusion problem for finite primitive permutation groups, University of Western Australia Research Report, 1987.

[10] A.D. Thomas and G.V. Wood, Group Tables (Shiva Publishing Limited, Devon Print Group, Exeter, 1980).

[11] H. Wielandt, Finite Permutation Groups (Academic Press, New York and London, 1964).

[12] D. Wright, 'Degrees of minimal embeddings for some direct products', Amer. J. Math. 97 (1976), $897-903$.

Dr. D. Easclown

Department of Mathematics,

The University of Western Australia, Nedlands, WA 6009

Australia

\section{Professor C.E. Praeger}

Department of Mathematics,

The University of Western Australia,

Nedlands, WA 6009

Australia 